Christian M. Meyer (Darmstadt)

\title{
Kollaborative Lexikografie: Strukturen, Dynamik und Zusammensetzung gemeinschaftlich erarbeiteter Wortschätze
}

\begin{abstract}
In diesem Beitrag diskutieren wir einen neuen Ansatz zur Erarbeitung von Wörterbüchern, bei dem eine große Zahl Freiwilliger gemeinsam ein OnlineWörterbuch entwickelt. Wir charakterisieren zunächst die wesentlichen Eckpunkte dieses nutzergetrieben-kollaborativen Vorgehens und diskutieren den aktuellen Stand der Forschung. Wir untersuchen Struktur, Dynamik und Zusammensetzung von kollaborativ erarbeiteten Wortschätzen und diskutieren, welches Innovationspotenzial in kollaborativen Wörterbüchern steckt und ob professionell erstellte Wörterbücher durch den neuen Ansatz obsolet werden.
\end{abstract}

\section{Einleitung}

Wörterbücher werden traditionell von wenigen Expertinnen und Experten erarbeitet. Deren Tätigkeit basiert auf Kenntnissen zu Lexikografie, Wörterbuchforschung, Sprachwissenschaft und Korpusanalyse, wie sie etwa im ErasmusMundus-Studiengang „Europäischer Master für Lexikographie“ (Schierholz 2010) und in der Einarbeitungsphase zu einem konkreten Wörterbuchprojekt vermittelt und entwickelt werden. Ziel dieser Ausbildung ist es, eine konsistente, qualitativ hochwertige und an den vorgesehenen Einsatzzweck angepasste Beschreibung eines Wortschatzes erarbeiten zu können.

Im Gegensatz zu diesem professionellen Ansatz hat sich in jüngerer Zeit ein neuer Ansatz der Wörterbucherstellung herausgebildet, bei dem eine große Zahl Freiwilliger maßgeblich ein Wörterbuch prägt: das Paradigma der kollaborativen Lexikografie. Eine spezifische Ausbildung ist zur Beteiligung nicht erforderlich, sodass auch Laien an einem Wörterbuch mitarbeiten können. Wir bezeichnen die Freiwilligen häufig als „Nutzer“, da die scheinbar klare Grenze zwischen Wörterbucherstellern und Wörterbuchbenutzern verschwimmt. Lew (2014) spricht von Prosumern, da die Rollen Produzent und Konsument zusammenfallen. Durch die vielen Beteiligten ergeben sich potenziell unterschiedliche Sichtweisen, die im Wörterbuch konsolidiert werden müssen. Die Wörterbuchinhalte und die Richtlinien zur Erarbeitung der lexikografischen Beschreibungen sind daher nicht 
mehr notwendigerweise statisch, sondern entwickeln sich evolutionär durch Überarbeitung, Diskussion und Abstimmung. Es entsteht ein gemeinschaftlich erarbeiteter Wortschatz einer Sprachgemeinde.

Im vorliegenden Beitrag nähern wir uns zunächst dem Begriff der kollaborativen Lexikografie, indem wir verschiedene Nutzerbeteiligungsformen diskutieren und den Stand der Forschung zusammenfassen. Anhang zweier Forschungsfragen gehen wir dann auf ausgewählte Aspekte zur Struktur, Dynamik und Zusammensetzung von gemeinschaftlichen Wortschätzen ein und schließen mit einigen Schlussfolgerungen und einem Ausblick.

\subsection{Nutzerbeteiligung bei Online-Wörterbüchern}

Benutzer an der Wörterbucherstellung zu beteiligen ist keine neue Idee. Bereits in den Anfangsjahren des „Oxford English Dictionary“ (damals noch „New English Dictionary“) hat die Philologische Gesellschaft Londons ab 1879 zur Einsendung von Belegen aufgerufen (siehe Thier 2014). Mit Etablierung des World Wide Web und der um die Jahrtausendwende aufgekommenen Technologien im sogenannten Web 2.0 haben sich jedoch völlig neue Möglichkeiten zur Nutzerbeteiligung ergeben.

Insbesondere war es vor Aufkommen des Webs nur schwer möglich, den aktuellen Bearbeitungsstand eines Wörterbuchs mit allen Benutzern auszutauschen, sodass sich diese lediglich indirekt beteiligen konnten (etwa durch postalische Einsendungen). Werden die lexikografischen Daten dagegen online zugänglich gemacht, beispielsweise auf kollaborativen Textproduktionsplattformen wie Blogs oder Wikis, so wird erstmals eine umfangreiche direkte Beteiligung möglich. Neben den neuen Interaktions- und Kommunikationsmöglichkeiten in Echtzeit, ist das Web auch zu einem Massenphänomen geworden. OnlineWörterbücher können daher sehr große Nutzerzahlen erreichen, deren Beiträge das Wörterbuch und auch die Beteiligungsformen selbst nachhaltig verändern können.

Abel/Meyer (2016) legen eine detaillierte Klassifikation der vielfältigen Möglichkeiten zur Beteiligung von Nutzern an Online-Wörterbüchern vor. Die wesentlichen Eigenschaften sind im Folgenden wiedergegeben und in Abbildung 1 zusammengefasst. 

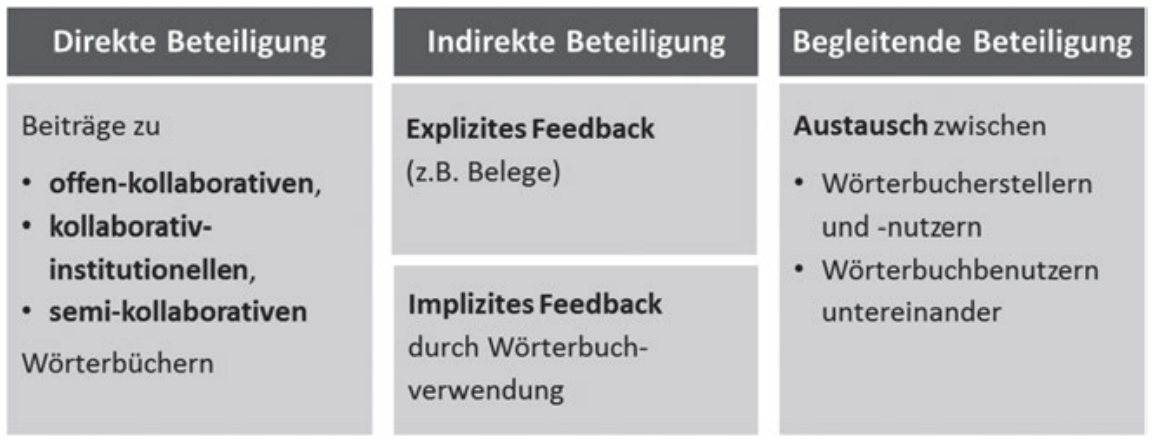

Abb. 1: Möglichkeiten zur Nutzerbeteiligung bei Online-Wörterbüchern (nach Abel/Meyer 2016)

Direkte Nutzerbeteiligungsformen erlauben es den Benutzern, selbstständig neue Wörterbucheinträge beizutragen. Diese Einträge werden entweder sofort und unverändert in das Wörterbuch übernommen oder nach einer Prüfung durch Moderatoren oder Herausgeber unverändert oder leicht revidiert freigeschaltet. Einige Wörterbuchprojekte ermöglichen zudem das Verändern und Löschen von eigenen oder fremden Beiträgen. Wörterbücher, die in hohem Maße eine direkte Nutzerbeteiligung ermöglichen, werden auch kollaborative Wörterbücher genannt und bilden den Schwerpunkt des kollaborativen Paradigmas und dieses Aufsatzes. Wir können drei wesentliche Varianten kollaborativer Wörterbücher unterscheiden:

1. Offen-kollaborative Wörterbücher, wie Wiktionary oder OmegaWiki, verfügen über keinen fest vorgegebenen Herausgeber. Nutzerbeiträge werden unmittelbar ins Wörterbuch übernommen und später von anderen Benutzern weiter bearbeitet, korrigiert oder gelöscht. Die Nutzer erarbeiten nicht nur die gesamten Einträge, sondern konzipieren die lexikografischen Instruktionen, organisieren die Zusammenarbeit und übernehmen die Qualitätskontrolle.

2. Kollaborativ-institutionelle Wörterbücher, wie das Merriam-Webster Open Dictionary und das Macmillan Open Dictionary werden von Verlagen oder Instituten angeboten und dienen der Bündelung von Nutzervorschlägen zur Erweiterung eines redaktionell gepflegten Wörterbuchs. Macmillan übernahm etwa die von Nutzern beigetragenen Beschreibungen zu hypocaust in das verlagseigene Online-Wörterbuch. ${ }^{1}$ Beigetragene Einträge werden vor Freischaltung lediglich auf groben Unfug und Spam überprüft, während die Beschreibungen unverändert bleiben und erst bei einer etwaigen Übernahme in andere Produkte evaluiert werden.

1 www.macmillandictionary.com/dictionary/british/hypocaust (Stand: 10.4.2017). 
1. Semi-kollaborative Wörterbücher wie die Wörterbücher auf LEO Online und das Synonymwörterbuch OpenThesaurus erlauben ebenfalls direkte Nutzerbeiträge. Diese werden eingehend geprüft und entweder in ein bestehendes Wörterbuch integriert oder abgewiesen.

Können Benutzer ein Wörterbuch dagegen nur mittelbar verändern, sprechen wir von indirekter Nutzerbeteiligung. Die Nutzerbeiträge werden gesammelt, von den Wörterbuchherausgebern revidiert und ergänzt, bevor sie in das Wörterbuch eingearbeitet werden. Wir unterscheiden explizites und implizites Feedback als zwei wesentliche Erscheinungsformen von indirekter Nutzerbeteiligung.

1. Unter explizites Feedback fassen wir Vorschläge für neue Stichwörter, Fehlerkorrekturen, Ergänzungen zu einzelnen Einträgen sowie allgemeine Verbesserungsvorschläge zum Wörterbuch. Wie eingangs ausgeführt, sind derartige Beiträge bereits aus Printwörterbüchern bekannt. Neben postalischen Einsendungen kommen in Online-Wörterbüchern vor allem Einsendungen per E-Mail oder Webformular hinzu. Als exemplarische Beispiele für Wörterbücher mit explizitem Feedback seien das Oxford English Dictionary und Duden online genannt.

2. Implizites Feedback wird von Benutzern nicht bewusst, sondern unbewusst durch deren Verwendung des Wörterbuchs erhoben. Dazu zählen Protokolldateien, die angeben, welche Wörterbucheinträge wann und wie oft angesehen wurden, benutzerbezogene Daten, wie persönliche Wortlisten, Studien zur Wörterbuchbenutzung sowie von Benutzern auf externen Plattformen beigetragene Daten (z.B. bei Kurznachrichtendiensten oder Fotoportalen).

Begleitende Nutzerbeteiligung bildet die dritte Klasse der Beteiligungsmöglichkeiten. Dabei geht es jedoch nicht um die direkte oder indirekte Veränderung der lexikografischen Beschreibungen, sondern um den Austausch zwischen Wörterbuchbenutzern und -herausgebern sowie zwischen Wörterbuchbenutzern selbst. Neben Blogs zu wörterbuchrelevanten Themen werden vor allem Diskussionsforen und Seiten mit Kommentarfunktion eingesetzt. Macmillan publiziert beispielsweise die regelmäßige Serie „Language tip of the week“ mit zahlreichen Links zu Wörterbucheinträgen und Nutzerkommentaren. ${ }^{2}$ Umfangreiche Diskussionsforen finden sich unter anderem bei LEO Online, wo Benutzer sich über die Wörterbuchinhalte hinaus zu Übersetzungen austauschen. Auch Sprachspiele zum Kennenlernen eines Wörterbuchs, etwa zum niederländischen „Algemeen Nederlands Woordenboek“ (Schoonheim et al. 2012) und professionelle Sprachbera-

2 www.macmillandictionaryblog.com/category/language-tips (Stand: 10.4.2017). 
tungsangebote, wie sie von Duden oder auf canoonet ${ }^{3}$ angeboten werden, zählen zu den begleitenden Nutzerbeteiligungsformen.

\subsection{Stand der Forschung}

Frühe Arbeiten zur kollaborativen Lexikografie gehen auf Carr (1997) und Storrer/ Freese (1996) zurück. Carr prägt den Begriff bottom-up lexicography, geht allerdings noch vorwiegend auf die Einsendung von Wörterbucheinträgen per E-Mail ein. Storrer (1998, 2013) diskutiert verschiedene Möglichkeiten zur Einsendung von Fehlerkorrekturen, Lemmavorschlägen und ganzen Wörterbucheinträgen von Laien oder Experten. Lew (2011) fasst Nutzerbeteiligung breiter und beschreibt verschiedene Grade der direkten Nutzerbeteiligung in englischsprachigen Online-Wörterbüchern.

$\mathrm{Zu}$ Wiktionary als größtem Vertreter kollaborativer Wörterbücher liegen die meisten Publikationen vor. Fuertes Olivera (2009), Hanks (2012) und Rundell (2012) gehen auf die Qualität der lexikografischen Angaben ein, Abel (2006) untersucht die Wörterbuchstrukturen, Matuschek/Meyer/Gurevych (2013) analysieren multilinguale Eigenschaften und Meyer (2013) gibt einen detaillierten Überblick zum lexikografischen Prozess und legt eine quantitative Auswertung vor, die wir im Folgenden aufgreifen. Über Wiktionary hinaus finden sich Arbeiten zu OmegaWiki (Meijssen 2009) und zu den semi-kollaborativen Projekten OpenThesaurus (Naber 2005) und LEO Online (Melchior 2012).

Auf indirekte und begleitende Nutzerbeteiligung gehen unter anderem de Schryver/Prinsloo (2000), Køhler Simonsen (2005) und Lew (2014) ein. Außerdem diskutierten Thier (2014) die Beteiligungsmöglichkeiten für das Oxford English Dictionary und Rautmann (2014) für Duden online. Die Schnittstelle zur Wörterbuchbenutzungsforschung ist bei Müller-Spitzer (Hg.) (2014) detailliert ausgeführt.

Abel/Meyer (2013, 2016) legen eine systematische Beschreibung zur Nutzerbeteiligung in Online-Wörterbüchern vor und diskutieren diese anhand zahlreicher Beispiele. Darauf aufbauend ist im Rahmen des European Network of e-Lexicography (ENeL) erstmals eine Erhebung zur Nutzerbeteiligung in Online-Wörterbüchern entstanden, die jüngst von Carole Tiberius und Bob Boelhouwer ausgewertet wurde. ${ }^{4}$ In ihrer aktuellen Arbeit benennen Meyer/Abel (2017) spezifische Forschungsfragen zur Planung, Umsetzung und Evaluation von Nutzerbeteiligung bei Online-Wörterbüchern.

$3 \mathrm{http} / /$ canoo.net/blog/ (Stand: 19.4.2017).

4 www.elexicography.eu/working-groups/working-group-3/wg3-meetings/wg3-barcelona-2016/ (Stand: 19.4.2017). 


\subsection{Forschungsfragen}

Obwohl die ersten Arbeiten zur kollaborativen Lexikografie und große Wörterbuchprojekte wie Wiktionary mittlerweile gut fünfzehn Jahre alt sind, ist die Erforschung des nutzergetriebenen Ansatzes nach wie vor aktuell und brisant. Im Bereich der Sachlexikografie hat die kollaborativ erstellte Enzyklopädie Wikipedia Verlagsprodukte wie die „Encyclopaedia Britannica“ oder die „BrockhausEnzyklopädie“ weitgehend verdrängt - oder zumindest Anteil an dieser Entwicklung. In der Sprachlexikografie verändern sich die Geschäftsmodelle derzeit stark (vgl. Kernerman (Hg.) 2013), sodass wir der Frage nachgehen, ob kollaborative Wörterbücher etablierte Verlags- oder Akademieprojekte verdrängen werden und welche Konsequenzen sich daraus für die Lexikografie ergeben. Neben der Frage zum zukünftigen Stellenwert der (kollaborativen) Lexikografie, diskutieren wir für ausgewählte Aspekte, welches Innovationspotenzial der kollaborative Ansatz mit sich bringt.

\section{Strukturen}

Kollaborative Wörterbücher nutzen überwiegend Strukturen, die aus professionell erarbeiteten Wörterbüchern bekannt sind. Auf der Ebene der Makrostruktur finden sich etwa alphabetische Wortlisten, verschiedene Suchfunktionen oder der Zugriff über Wort-/Themenfelder. Eine Mediostruktur mit unterschiedlichen Verweistypen wird mittels Hyperlinks erreicht, die beispielsweise Übersetzungsäquivalente, sinnverwandte oder syntaktisch ähnliche Stichwörter vernetzen. Auch auf der Ebene der Mikrostruktur finden wir eine große Bandbreite an Angabeklassen und Darstellungsformen, die sich gleichermaßen in professionellen und kollaborativen Projekten finden.

Gerade im Bereich der sprachübergreifenden Lexikografie weisen kollaborative Projekte jedoch interessante Vernetzungsstrukturen auf, die wir im Folgenden näher diskutieren. Während beim professionellen Vorgehen schon allein aus Personalgründen ein- und zweisprachige Angebote im Vordergrund stehen, finden sich zahlreiche kollaborative Projekte mit deutlich mehr als zwei Sprachen. Wir betrachten die in Abbildung 2(a) schematisch dargestellte Situation: Ein Eintrag L1:E1 der Sprache L1 hat zwei Übersetzungsäquivalente L2:E1 und L2:E2 in Sprache L2 und zwei Äquivalente in einer weiteren Sprache L3. L2:E1 kann als L3:E1 in L3 repräsentiert werden, ist jedoch kein passendes Äquivalent von L3:E2. Ebenso ist die Übersetzung L2:E2-L3:E2 passend, L2:E2-L3:E1 aber nicht. 


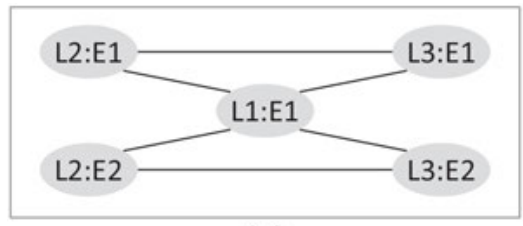

(a)

\begin{tabular}{|c|c|c|}
\hline L1 & L2 & L3 \\
\hline L1:E1 & L2:E1 / L2:E2 & L3:E1 / L3:E2 \\
\hline
\end{tabular}

(c)

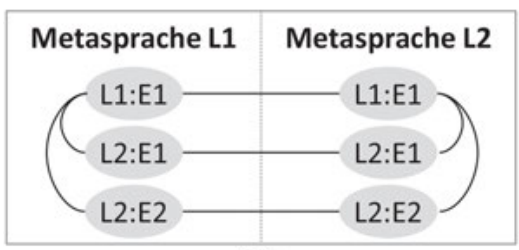

(e)

\begin{tabular}{|c|c|c|c|c|c|c|}
\hline L1 & L2 \\
\hline L1:E1 & L2:E1 & L3 \\
\hline L1: & L2 & L3 \\
\hline L1:E1 & L2:E2 2 L3:E1 \\
\hline L1:E1 & L3:E2 2 \\
\hline L2:E1 & L3:E1 \\
\hline L2:E2 & L3:E2 \\
\hline
\end{tabular}

(b)

\section{$\{$ L1:E1, L2:E2, L3:E2 \}}

(d)

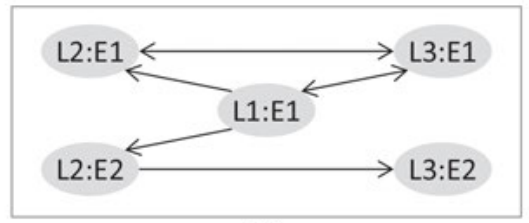

(f)

Abb. 2: Vernetzung und Präsentation von Übersetzungsäquivalenten in mehrsprachigen kollaborativen Wörterbüchern

Eine naheliegende Struktur zur Speicherung und Darstellung von Mehrsprachigem ist die Integration vieler zweisprachiger Angebote in einem Wörterbuchportal. Das ist beispielsweise auf LEO Online oder dict.cc der Fall und auch gängige Praxis in der Verlagslexikografie (etwa bei PONS). Übersetzungsäquivalente werden für je ein Sprachpaar tabellarisch aufgelistet. Hat ein Stichwort bzw. eine Bedeutung der Quellsprache mehrere Äquivalente in der Zielsprache, wird es für jedes Äquivalent wiederholt. Abbildung 2(b) zeigt eine solche Darstellung für die drei Sprachen aus Abbildung 2(a). Ein Nachteil dieser Struktur ist, dass die Beziehungen der einzelnen Sprachpaare untereinander nicht direkt sichtbar sind. Wird eine Übersetzung für L1:E1 in L2 gesucht (linke Tabelle), so könnten die jeweiligen Äquivalente in L3 bei der Wahl der passenden Übersetzung helfen, sofern ein Benutzer auch L3 versteht. Die paarige Struktur würde dazu jedoch erfordern, dass mindestens das L2-L3-Wörterbuch zusätzlich aufgerufen werden muss.

Das kollaborative „Refugee Phrasebook“ ist ein benutzergruppenorientiertes mehrsprachiges Wörterbuch, das häufig genutzte Wendungen in kommunikativen Situationen von Geflüchteten in knapp 60 Sprachen sammelt. Das Projekt besteht 
seit 2015 und wird von der Open Knowledge Foundation ${ }^{5}$ unterstützt. Die im Wörterbuch gespeicherten Wendungen werden von Nutzern beigetragen, nach Themen geordnet und in einer einzigen großen Tabelle rechtefrei im Internet zugänglich gemacht. Neben Interjektionen zur Begrüßung und Verabschiedung finden sich (Teil-)Sätze zur Beschreibung von Symptomen oder Erkrankungen (z.B. Ich habe Fieber) mit passenden Vokabeln oder Rechtsthemen (z.B. Ich möchte die Botschaft meines Landes kontaktieren). Jede Zeile der Tabelle gibt für eine Wendung die Äquivalente in den 60 Sprachen an. Abbildung 2(c) zeigt diese Struktur schematisch. Zwar können auf diese Weise Äquivalente in mehreren Sprachen auf einmal dargestellt werden, allerdings bringt die feste Zeilenstruktur den Nachteil mit sich, dass Mehrdeutigkeiten und Übersetzungsvarianten (z.B. Spanisch gata/ gato für Katze) in einer einzigen Tabellenzelle angegeben und kommentiert werden müssen. Ohne eine solche Kommentierung könnte wiederum kaum darauf geschlossen werden, dass L2:E1-L3:E2 keine passende Übersetzung ist.

Wiktionary wählt einen weiteren Ansatz, indem einerseits je Wortbedeutung die Äquivalente in sämtlichen Sprachen aufgelistet werden und andererseits alle objektsprachlichen Angaben in mehreren Metasprachen beschrieben werden können. Im deutschen Wiktionary findet sich etwa der Eintrag Maus mit Übersetzungen in 51 Sprachen sowie der Eintrag zum englischen mouse, welcher neben den Übersetzungen in 4 Sprachen auch auf den entsprechen Eintrag im englischen Wiktionary verweist. Die Angaben im deutschen Wiktionary sind dabei generell auf Deutsch formuliert (auch für mouse), während das englische Wiktionary ausschließlich englische Beschreibungen enthält. Übersetzungsäquivalente sind daher sowohl in der Quellsprache als auch in der Zielsprache beschrieben, wodurch ein komplexes Linknetzwerk entsteht. Abbildung 2(e) zeigt einen Ausschnitt für L1 und L2.

Die hohe Redundanz dieses Ansatzes kann jedoch leicht zu Inkonsistenzen führen. Wird eine neue Übersetzung von L1 in L2 eingetragen, so müsste dies ebenso in den fremdsprachlichen Einträgen aller Sprachversionen sowie in jeweils umgekehrter Richtung von L2 nach L1 in allen Sprachversionen geändert werden. Da die Bedeutungsbeschreibungen in jeder Sprachversion voneinander abweichen können, ist dies womöglich nicht ohne weitere Änderung möglich (z.B. da die entsprechende Bedeutung noch gar nicht gelistet ist). Abbildung 2(f) zeigt ein Beispiel für eine solche lückenhafte Struktur.

In OmegaWiki findet sich wiederum eine andere Struktur. Bedeutungsgleiche Wortbedeutungen werden zu sprachübergreifenden Konzepten gruppiert (bei OmegaWiki sogenannte DefinedMeanings). Diese Struktur findet sich bisher vor

5 https://okfn.de/ (Stand: 9.6.2017). 
allem in mehrsprachigen Wortnetzen in der Computerlinguistik, siehe etwa Vossen (1998) zu EuroWordNet. Für die Bedeutung des Nagetiers Maus sind beispielsweise 130 Einträge in 118 Sprachen vermerkt. In einer solchen Struktur kann etwa L1:E1, wie in Abbildung 2(d) gezeigt, zu zwei Konzepten zugeordnet werden, ohne die Einträge L2:E1 und L3:E2 als valide Übersetzungen zu kennzeichnen.

\section{Dynamik}

Die inhaltliche Untersuchung und Bewertung von Wörterbüchern ist Teil von Wörterbuchkritiken. Diese wählen in der Regel einzelne Einträge zur Diskussion aus, beispielsweise 50 zufällige Adjektive oder 20 aufeinanderfolgende Druckseiten (Chan/Taylor 2001). Dieses Vorgehen eignet sich gut für professionell erarbeitete Wörterbücher, da erwartet werden kann, dass die gewählten Einträge fertig bearbeitet sind und den lexikografischen Instruktionen genügen.

Kollaborative Wörterbücher sind grundsätzlich Ausbauwörterbücher. Sie werden also nicht in Ergänzungslieferungen oder als vollständige Neuauflage veröffentlicht, sondern den Benutzern direkt zur Bearbeitung online zugänglich gemacht. Es ist dementsprechend schwer zu sagen, wann ein Eintrag fertig ist. Nicht selten sind neu angelegte Einträge zunächst unvollständig oder fehlerhaft. Erst mit der Zeit werden die rudimentären Angaben von anderen Benutzern ergänzt und revidiert, wodurch sich ein Eintrag jederzeit stark verändern kann. Das Verb zufriedenstellen zum Beispiel wurde 2007 im deutschen Wiktionary mit lediglich einer Bedeutungsparaphrase und drei englischen Übersetzungen beschrieben. Im Verlauf eines Jahres sind zahlreiche Angabeklassen, etwa zur Flexion und Aussprache hinzugekommen, die den Artikel vorläufig komplettierten. Die aktuell vorliegende Fassung vom Oktober 2016 ist aber das Ergebnis weiterer substanzieller Überarbeitungen und weist unter anderem zusätzliche Beispielangaben, eine neu formulierte Bedeutungsparaphrase und Angaben zu Synonymen und Antonymen auf. Der Eintrag, an dem 21 verschiedene Autoren mitgewirkt haben, wurde insgesamt 39 Mal überarbeitet.

Da Inhalt und Qualität eines einzelnen Eintrags also stark vom Auswertungszeitpunkt abhängen, müssen wir die verschiedenen Bearbeitungsstände in kollaborativen Wörterbüchern in die Analyse einbeziehen. Stichproben können entweder gezielt aus Einträgen mit vielen Überarbeitungen oder frühem Erstellungszeitpunkt gebildet werden oder das Wörterbuch wird mithilfe quantitativer Verfahren als Ganzes betrachtet. Um einen fairen Vergleich mehrerer Wörterbücher zu ermöglichen, eignen sich standardisierte Lexikonmodelle wie Lexical Markup Framework (LMF; siehe Francopoulo (Hg.) 2013), Text Encoding Initiative (TEI; 
siehe z.B. Declerck/Mörth/Lendvai 2012) oder lemon (McCrae/Spohr/Cimiano 2011). Eckle-Kohler et al. (2012) schlagen mit UBY-LMF ein auf LMF basierendes Lexikonmodell vor, mit dem auch kollaborative Wörterbücher abbildbar sind. Wir nutzen dieses Lexikonmodell für die nachfolgenden Auswertungen.

Tab. 1: Wachstum und Benutzerzahlen im deutschen Wiktionary

\begin{tabular}{lllll}
\hline Jahr & $\begin{array}{l}\text { Gesamtzahl } \\
\text { der Einträge }\end{array}$ & $\begin{array}{l}\text { Neue Einträge } \\
\text { pro Tag }\end{array}$ & $\begin{array}{l}\text { Registrierte } \\
\text { Benutzer }\end{array}$ & Aktive Benutzer \\
\hline 2017 & 592.000 & 242 & 1.488 & 59 \\
2016 & 464.000 & 365 & 1.385 & 70 \\
2015 & 387.000 & 188 & 1.241 & 71 \\
2014 & 344.000 & 116 & 1.143 & 61 \\
2013 & 230.000 & 311 & 1.026 & 61 \\
2012 & 203.000 & 66 & 929 & 53 \\
2011 & 141.000 & 203 & 841 & 56 \\
2010 & 103.000 & 75 & 724 & 57 \\
2009 & 88.000 & 41 & 603 & 57 \\
2008 & 72.000 & 51 & 475 & 53 \\
2007 & 46.000 & 66 & 350 & 53 \\
2006 & 18.000 & 77 & 204 & 49 \\
2005 & 3.900 & 36 & 78 & 32 \\
\hline
\end{tabular}

Tabelle 1 zeigt die Zahl der Wörterbucheinträge im deutschen Wiktionary zu Beginn des jeweiligen Jahres sowie die durchschnittliche Zahl der neu angelegten Einträge pro Tag. ${ }^{6}$ Kollaborative Wörterbücher können offensichtlich schnell wachsen, doch sollte die Zahl der Einträge nicht überbewertet werden, da rund 49\% der Einträge flektierte Wortformen und 31\% fremdsprachliche Stichwörter beschreiben.

Die Zahl der registrierten und durchschnittlich aktiven Benutzer ist ebenfalls in der Tabelle angegeben. Registrierte Benutzer haben sich für ein Benutzerkonto angemeldet und leisten den überwiegenden Teil der Beiträge. Dazu kommen rund 3\% anonyme Beiträge von Nutzern, die sich nicht registriert haben und Beiträge, die automatisiert durch von Nutzern programmierte Bots erfolgen. Zu den aktiven Benutzern zählen registrierte Benutzer, die innerhalb eines Monats mindestens fünf Beiträge geliefert haben. Deren Anzahl wird für jeden Monat erfasst und über je ein Jahr gemittelt. Während die Zahl der registrierten Benutzer nahezu

6 https://stats.wikimedia.org/wiktionary/EN/TablesWikipediaDE.htm (Stand: 20.4.2017). 
gleichmäßig um rund 10-20 Benutzer pro Monat wächst, nimmt die Zahl der aktiven Benutzer nur leicht zu. Auch ist diese Kerngruppe großen Schwankungen unterworfen, da sehr aktive Benutzer eines Monats nicht notwendigerweise auch im Folgemonat noch aktiv sind. Die Nutzerbeiträge sind insgesamt stark unterschiedlich verteilt: Ein Großteil der registrierten Benutzer nimmt nur eine oder überhaupt keine Änderung vor, während es wenige Benutzer gibt, die sehr viele Beiträge liefern. Diese Art der Verteilung ist charakteristisch für Online-Gemeinschaften (siehe Rafaeli/Ariel 2008).

Das deutsche Wiktionary ist damit ein Produkt einer rund 60-köpfigen Kerngruppe, deren Mitgliederzahl sich über die Zeit hinweg verändert, und von über 1.400 weiteren Beitragenden. Das englische Wiktionary ist mit 5,1 Millionen Einträgen (davon über 700.000 zu englischen Stichwörtern), 7.700 registrierten Benutzern und durchschnittlich 300 aktiven Benutzern deutlich größer, weist aber eine ähnliche Verteilung auf.

Häufig übernehmen Benutzer eine bestimmte Rolle, beispielsweise das Ergänzen von Übersetzungsäquivalenten einer bestimmten Sprache, das Bearbeiten von Stichwörtern einer Fachdomäne, die einheitliche Formatierung der Einträge oder das Entfernen von Vandalismus und Spam. Meyer/Gurevych (2014) werten die Beiträge einzelner Benutzer eingehender aus und berichten, dass rund ein Drittel der Überarbeitungen eines Eintrags von je einem Benutzer durchgeführt werden, der für eine gewisse Zeit eine Art Patenschaft für den Eintrag übernimmt und in dieser Zeit die Beiträge weiterer Freiwilliger konsolidiert.

Insbesondere in offen-kollaborativen Wörterbüchern verändern sich nicht nur die Wörterbucheinträge, sondern auch die lexikografischen Instruktionen. Zwar finden größere Änderungen vor allem zu Beginn eines Projekts statt, aber auch nach der Planungsphase werden noch umfangreiche Anpassungen vorgenommen. Das englische Wiktionary diskutiert aktuell beispielsweise, ob Synonymangaben den einzelnen Bedeutungen zugeordnet werden sollen, statt diese in einem eigenen Abschnitt von den Bedeutungsbeschreibungen separiert anzugeben. ${ }^{7}$

Die dynamische Veränderung von Einträgen und Instruktionen in kollaborativen Wörterbüchern wird von bekannten Prozessmodellen in der professionellen Lexikografie kaum widergespiegelt. Meyer (2013) entwirft daher ein zyklisches Prozessmodell für das Beispiel Wiktionary, in dem wiederholte Änderungen an Instruktionen und Einträgen sowie die kollaborativen Diskussionsprozesse abgebildet werden (siehe Abb. 3).

7 https://en.wiktionary.org/wiki/Wiktionary:Beer_parlour/2017/February\#Placement_of_ synonyms (Stand: 12.4.2017). 


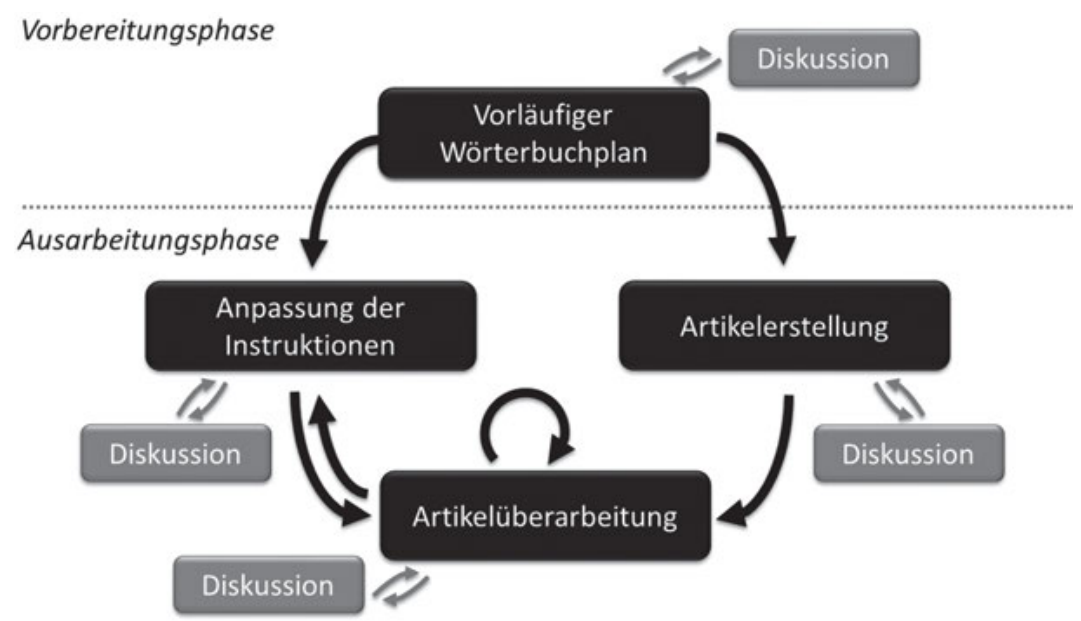

Abb. 3: Der lexikografische Prozess in Wiktionary (Meyer 2013)

\section{Zusammensetzung}

Abschließend betrachten wir die inhaltliche Zusammensetzung gemeinschaftlich erarbeiteter Wortschätze. Wir vergleichen dazu die kollaborativen Projekte Wiktionary und OpenThesaurus mit dem Tübinger Wortnetz GermaNet (Kunze/Lemnitzer 2002) in ihren jeweiligen UBY-LMF Repräsentationen. ${ }^{8}$ Eine detaillierte Analyse für ältere Datenbestände liegt bei Meyer/Gurevych (2012) vor, die auch eine kontrastive Untersuchung für Englisch und Russisch diskutieren.

Um Teilwortschätze zu identifizieren, die in kollaborativen Wörterbüchern über- oder unterrepräsentiert sind, gleichen wir zunächst automatisiert die Stichwortlisten der drei Wörterbücher ab. Einträge zu flektierten Wortformen sowie fremdsprachliche Einträge in Wiktionary werden dabei ignoriert, um die Vergleichbarkeit der Stichwortlisten zu gewährleisten. Abbildung 4 zeigt ein VennDiagramm, das die Zahl der Stichwörter je Wörterbuch sowie die jeweiligen Überschneidungen mit den übrigen Wörterbüchern zeigt. Eine zunächst überraschende Einsicht ist, dass sich nur rund ein Fünftel der Stichwörter eines Wörterbuchs in allen drei Quellen finden lassen und die überwiegende Mehrheit sogar nur in einem Wörterbuch beschrieben wird.

8 Ausgewertet werden die Datenbestände vom 1.2.2017 (deutsches Wiktionary) und vom 5.3.2017 (OpenThesaurus) sowie GermaNet 9.0. 


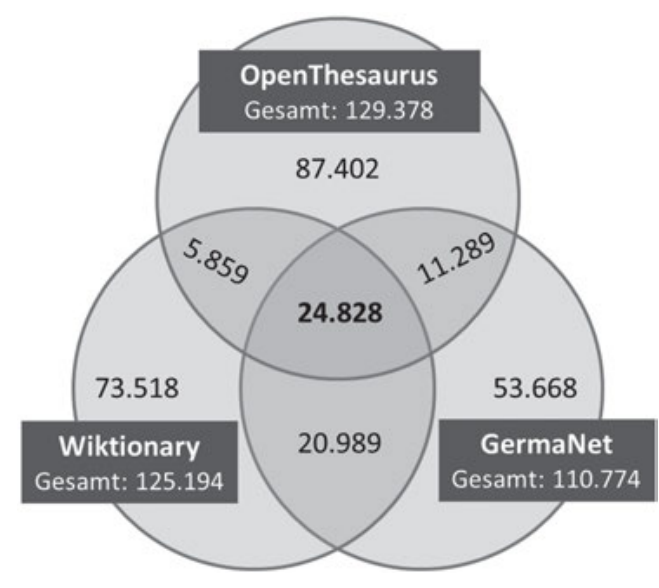

Abb. 4: Venn-Diagramm zur Anzahl der Stichwörter und wie viele davon sich in nur einem, zwei oder allen drei Wörterbüchern finden

Stichwörter, die nur in je einem der drei Wörterbücher belegt sind, haben wir näher untersucht, um charakteristische Teilwortschätze identifizieren zu können. Neben Berufsbezeichnungen (z.B. Altenpflegehelferin, Erzkanzler, Mälzerin) und Zahlwörtern (100-fach, achtspurig, elfbändig) finden sich in GermaNet zahlreiche Verben, die in keiner der beiden kollaborativen Quellen belegt sind, darunter banalisieren, fertig bekommen, lasten, rümpfen. Etwa 8,6\% der Stichwörter in GermaNet sind Verben, jedoch nur rund 5,6\% in Wiktionary. In der 7. Auflage des Duden Universalwörterbuch (2011, S. 2111) sind 11,1\% der Einträge Verben, was den Unterschied noch verdeutlicht. Verben sind häufig schwierig zu beschreiben und hochgradig polysem, was ein Grund für die Diskrepanz sein kann.

In Wiktionary findet sich dagegen eine große Zahl neu aufgekommener oder in aktuellen Diskussionen geprägte Stichwörter, zum Beispiel Flexirente, Helikoptereltern, Inklusionsklasse, Schmähgedicht sowie über 90 Komposita mit Erstglied Flüchtling, die sich in keiner der beiden anderen Quellen finden (z.B. Flüchtlingsflut, Flüchtlingsquote). Beide kollaborativen Projekte decken außerdem eine große Zahl an Fachtermen aus unterschiedlichsten Disziplinen ab - Beispiele sind Adjektivphrase (Sprachwissenschaft), Gichtgas (Montanindustrie), Grafiktreiber (Informatik), Pandektistik (Rechtswesen), Qualitätsopfer (Schach) - sowie umgangssprachliche und regionale Wendungen, wie Asi-Toaster (Solarium), Kneipenkaiser (Angeber), Lauschlappen (Ohren), Schnippelparty (Foodsharing-Aktion), Schrapphals (Ruhrgebiet: Geizhals). Die Vielfalt an solchen Nichtstandardvarietäten lässt sich auch quantitativ durch Auswertung der pragmatischen Markierungen belegen (siehe Meyer 2013). 
Betrachten wir nun die eigentlichen lexikografischen Beschreibungen, stellt sich die Frage nach Qualität und Quellen der Angaben. In professionell erarbeiteten Wörterbüchern werden Angaben überwiegend auf der Basis großer Textkorpora erarbeitet. Die Auswahl geeigneter Korpora ist zentraler Bestandteil der Wörterbuchplanung. In kollaborativen Projekten werden dagegen keine Korpora vorgegeben. Dementsprechend finden sich in Wiktionary zahlreiche Beispielangaben aus unterschiedlichen Quellen - insbesondere Online-Nachrichten und Fachliteratur - oder die Angaben bleiben ganz ohne Quellenangabe, z.B. wenn Beispiele selbst ausgedacht sind. Eine systematische Analyse von Korpora findet nicht statt und ist auch in den lexikografischen Instruktionen nicht beschrieben.

Stattdessen finden sich in den Wörterbucheinträgen auch zahlreiche Verweise auf andere Wörterbücher, deren Angaben offenbar in die Wörterbucharbeit einfließen und auch bei unterschiedlichen Sichtweisen häufig zur Schlichtung benannt werden. Wiktionary bietet zu diesem Zweck eine eigene Diskussionsseite für jeden Eintrag, auf der unterschiedliche Wahrnehmungen zum Sprachgebrauch diskutiert und konsolidiert werden können. Diese subjektiven Überlegungen zum Sprachgebrauch sind eine interessante Ergänzung zur systematischen Korpusanalyse, gerade wenn - wie im Fall von gesprochener Sprache, Umgangssprache, Briefen und privaten Chats usw. - nur wenige Korpora zur Verfügung stehen.

\section{Schlussfolgerungen und Ausblick}

Wenn wir nun auf die eingangs aufgeworfenen Forschungsfragen zurückkommen, stellen wir fest, dass kollaborative Wörterbücher anders entstehen als professionell erarbeitete Wörterbücher. Kollaborativ erarbeitete Angaben basieren auf Introspektion und dem Zusammentragen und Konsolidieren von Information aus unterschiedlichen, nicht fest vorgegebenen Quellen und bestehenden Wörterbüchern. Anders als beim professionellen Vorgehen findet keine systematische Korpusanalyse statt. Kollaborative Wörterbücher sind dementsprechend kein adäquater Ersatz für professionell erarbeitete Wörterbücher, sondern führen zu einem anderen Resultat, das zumindest teilweise subjektive Sprachwahrnehmungen wiedergibt.

Dies birgt vor allem Potenzial für Sprachvarietäten, die von bestehenden Korpora oder Wörterbüchern nur begrenzt oder zeitverzögert abgedeckt werden, wie Umgangssprache, Dialekte, Neuschöpfungen und Fachsprachen. Ferner profitiert die multilinguale Lexikografie von den vielen Beteiligten, da auch Übersetzungsäquivalente für Sprachpaare gesammelt werden, die kaum von kommerziellem Interesse sind. Andererseits zeigen sich auch Teilwortschätze, die in kollaborativen Wörterbüchern weniger gut abgebildet sind, beispielsweise Verben. 
Dabei gilt jedoch zu bedenken, dass eine Auswertung allenfalls eine Momentaufnahme liefert, da kollaborative Wörterbücher evolutionär entstehen und sich die Einträge über die Zeit stark verändern können oder neu entstehen.

Großes, bisher nur teilweise erschlossenes Potenzial sehen wir in der Zusammenarbeit von Freiwilligen und professionellen Lexikografinnen und Lexikografen, wenn die unterschiedlichen Herangehensweisen kombiniert werden. Einerseits ist das zwar genau der Weg, den beispielsweise das „Oxford English Dictionary“ seit vielen Jahren geht, andererseits gibt es in professionellen Wörterbüchern bisher jedoch kaum Maßnahmen, um die Arbeit der Freiwilligen über die Form der Eingaben hinaus zu systematisieren. Neben der Bereitstellung von Stichwortlisten könnten Quellen und Instruktionen zur Auswertung und Beschreibung in zahlreichen kleinen Aufgaben vorgegeben werden, die - wie bei Crowdsourcingplattformen - von ggf. mehreren Freiwilligen übernommen werden können. Dadurch könnte der Ausbau eines (kollaborativen oder professionellen) Wörterbuchs weniger interessen- und aktualitätsgesteuert, sondern systematisch nach einem Wörterbuchplan ablaufen und würde die Konsolidierung verschiedener subjektiver Sprachwahrnehmungen fördern. Eine wesentliche Herausforderung wird sein, diese Aufgaben überschaubar und interessant $\mathrm{zu}$ halten und zur Durchführung nötige Kenntnisse zu vermitteln. Mittelfristig kann dies für professionelle Wörterbücher auch zu einer stärkeren Nutzerbindung und zu gut ausgeprägten Benutzungskompetenzen führen.

\section{Literatur}

\section{Wörterbücher}

Algemeen Nederlands Woordenboek. Internet: http://anw.inl.nl.

Brockhaus Enzyklopädie. Internet: www.brockhaus.de/de/enzyklopaedie

dict.cc. Internet: www.dict.cc.

Duden (2011): Deutsches Universalwörterbuch. 7., überarb. u. erw. Aufl. Mannheim/Zürich.

Duden online. Internet: www.duden.de.

Encyclopædia Britannica. Internet: www.britannica.com.

LEO Online. Internet: http://dict.leo.org.

Macmillan Open Dictionary. Internet: www.macmillandictionary.com/open-dictionary.

Merriam-Webster Open Dictionary. Internet: http://nws.merriam-webster.com/opendictionary.

OmegaWiki. Internet: www.omegawiki.org.

OpenThesaurus. Internet: www.openthesaurus.de.

Oxford English Dictionary. Internet: www.oed.com.

Wikipedia. Internet: www.wikipedia.org.

Wiktionary. Internet: www.wiktionary.org. 


\section{Wissenschaftliche Literatur}

Abel, Andrea (2006): Elektronische Wörterbücher: Neue Wege und Tendenzen. In: San Vicente, Félix (Hg.): Lessicografia bilingue e traduzione: metodi, strumenti, approcci attuali. (= Lexicography Worldwide). Monza/Milano, S. 35-55.

Abel, Andrea/Klosa, Annette (Hg.) (2014): Der Nutzerbeitrag im Wörterbuchprozess. 3. Arbeitsbericht des wissenschaftlichen Netzwerks „Internetlexikografie“. (= OPAL. Online publizierte Arbeiten zur Linguistik 4/2014). Mannheim.

Abel, Andrea/Meyer, Christian M. (2013): The dynamics outside the paper: user contributions to online dictionaries. In: Kosem, Iztok et al. (Hg.): Electronic lexicography in the 21st century: Thinking outside the paper. Proceedings of the 3rd eLex Conference. Ljubljana/Tallinn, S. 179-194.

Abel, Andrea/Meyer, Christian M. (2016): Nutzerbeteiligung. In: Klosa, Anette/Müller-Spitzer, Carolin (Hg.): Internetlexikografie. Ein Kompendium. Berlin/New York, S. 249-290.

Bergenholtz, Henning/Nielsen, Sandro/Tarp, Sven (Hg.) (2009): Lexicography at a crossroads. Dictionaries and encyclopedias today, lexicographical tools tomorrow. (= Linguistic Insights. Studies in Language and Communication 90). Bern u.a.

Calzolari, Nicoletta et al. (Hg.) (2012): Proceedings of the 8th International Conference on Language Resources and Evaluation (LREC). Istanbul.

Carr, Michael (1997): Internet dictionaries and lexicography. In: International Journal of Lexicography 10, 3, S. 209-230.

Chan, Alice Yin Wa/Taylor, Andrew (2001): Evaluating learner dictionaries: What the reviews say. In: International Journal of Lexicography 14, 3, S. 163-180.

Declerck, Thierry/Mörth, Karlheinz/Lendvai, Piroska (2012): Accessing and standardizing Wiktionary lexical entries for the translation of labels in cultural heritage taxonomies. In: Calzolari (Hg.), S. 2511-2514.

Eckle-Kohler, Judith et al. (2012): UBY-LMF - a uniform model for standardizing heterogeneous lexical-semantic resources in ISO-LMF. In: Calzolari (Hg.), S. 275-282.

Fjeld, Ruth Vatvedt/Torjusen, Julie Matilde (Hg.) (2012): Proceedings of the 15th EURALEX International Congress. Oslo.

Francopoulo, Gil (Hg.) (2013): LMF. Lexical Markup Framework. London.

Fuertes Olivera, Pedro A. (2009): The function theory of lexicography and electronic dictionaries: Wiktionary as a prototype of collective free multiple-language internet dictionary. In: Bergenholtz/Nielsen/Tarp (Hg.), S. 99-134.

Granger, Sylviane/Paquot, Magali (Hg.) (2012): Electronic lexicography. Oxford.

Hanks, Patrick (2012): Word meaning and word use: Corpus evidence and electronic lexicography. In: Granger/Paquot (Hg.), S. 57-82.

Kernerman, Ilan (Hg.) (2013): Kernerman dictionary news 21. Tel Aviv.

Køhler Simonsen, Henrik (2005): User involvement in corporate LSP intranet lexicography. In: Gottlieb, Henrik/Mogensen, Jens Erik/Zettersten, Arne (Hg.): Symposium on Lexicography $\mathrm{XI}$. Proceedings of the Eleventh International Symposium on Lexicography. Tübingen, S. 489-510.

Kunze, Claudia/Lemnitzer, Lothar (2002): GermaNet - representation, visualization, application. In: Proceedings of the 3rd International Conference on Language Resources and Evaluation. Las Palmas, S. 1485-1491.

Lew, Robert (2011): Online dictionaries of English. In: Fuertes-Olivera, Pedro A./Bergenholtz, Hennig (Hg.): e-Lexicography. The internet, digital initiatives and lexicography. London/ New York, S. 230-250. 
Lew, Robert (2014): User-generated content (UGC) in English online dictionaries. In: Abel/Klosa (Hg.), S. 7-25.

Matuschek, Michael/Meyer, Christian M./Gurevych, Iryna (2013): Multilingual knowledge in aligned Wiktionary and OmegaWiki for translation applications. In: Translation: Computation, corpora, cognition: Special Issue on language technologies for a multilingual Europe 3, 1, S. 87-118.

McCrae, John/Spohr, Dennis/Cimiano, Philipp (2011): Linking lexical resources and ontologies on the semantic web with lemon. In: The semantic web: Research and applications. 8th Extended Semantic Web Conference (ESWC). (= Lecture Notes in Computer Science (LNCS) 6643). Berlin/Heidelberg, S. 245-259.

Meijssen, Gerard (2009): The philosophy behind OmegaWiki and the visions for the future. In: Bergenholtz/Nielsen/Tarp (Hg.), S. 91-98.

Melchior, Luca (2012): Halbkollaborativität und Online-Lexikographie. Ansätze und Überlegungen zu Wörterbuchredaktion und Wörterbuchforschung am Beispiel LEO Deutsch-Italienisch. In: Lexicographica 28, 1, S. 337-372.

Meyer, Christian M. (2013): Wiktionary. The metalexicographic and natural language processing perspective. (= TUprints 3654). Darmstadt. [Dissertation]. Internet: http://tuprints.ulb. tu-darmstadt.de/3654/.

Meyer, Christian M./Abel, Andrea (2017): User participation in the era of the internet. In: Fuertes Olivera, Pedro A. (Hg.): The Routledge handbook of Lexicography. Abingdon/New York, S. 735-753.

Meyer, Christian M./Gurevych, Iryna (2012): Wiktionary: A new rival for expert-built lexicons? Exploring the possibilities of collaborative lexicography. In: Granger/Paquot (Hg.), S. 259-291.

Meyer, Christian M./Gurevych, Iryna (2014): Methoden bei kollaborativen Wörterbüchern. In: Lexicographica 30, 1, S. 187-212.

Müller-Spitzer, Carolin (Hg.) (2014): Using online dictionaries. (= Lexicographica. Series Maior 145). Berlin/Boston.

Naber, Daniel (2005): OpenThesaurus: ein offenes deutsches Wortnetz. In: Fisseni, Bernhard et al. (Hg.): Sprachtechnologie, mobile Kommunikation und linguistische Ressourcen. Beiträge zur GLDV-Tagung 2005 in Bonn. (= Sprache, Sprechen und Computer/Computer Studies in Language and Speech 8). Frankfurt a.M., S. 422-433.

Rafaeli, Sheizaf/Ariel, Yaron (2008): Online motivational factors: Incentives for participation and contribution in Wikipedia. In: Barak, Azy (Hg.): Psychological aspects of cyberspace: Theory, research, applications. Cambridge, S. 243-267.

Rautmann, Karin (2014): Duden online und seine Nutzer. In: Abel/Klosa (Hg.), S. 48-61.

Rundell, Michael (2012): ,It works in practice but will it work in theory?` The uneasy relationship between lexicography and matters theoretical. In: Fjeld/Torjusen (Hg.), S. 47-92.

Schierholz, Stefan J. (2010): EMLex: Europäischer Master für Lexikographie - European Master in Lexicography. In: Lexicographica 26, 1, S. 343-350.

Schoonheim, Tanneke et al. (2012): Dictionary use and language games: getting to know the dictionary as part of the game. In: Fjeld/Torjusen (Hg.), S. 974-979.

de Schryver, Gilles-Maurice/Prinsloo, Daan J. (2000): Dictionary-making process with ,simultaneous feedback' from the target users to the compilers. In: Proceedings of the 9th EURALEX International Congress, Stuttgart, S. 197-209.

Storrer, Angelika (1998): Hypermedia-Wörterbücher: Perspektiven für eine neue Generation elektronischer Wörterbücher. In: Wiegand, Herbert Ernst (Hg.): Wörterbücher in der Diskussion III. Vorträge aus dem Heidelberger Lexikographischen Kolloquium. (= Lexicographica. Series Maior 84 ). Tübingen, S. 107-135. 
Storrer, Angelika (2013): Representing (computational) dictionaries in hypertextual form. In: Gouws, Rufus H. et al. (Hg.): Dictionaries. An international encyclopedia of Lexicography. Supplementary volume: Recent developments with focus on electronic and computational lexicography. (= Handbücher zur Sprach- und Kommunikationswissenschaft/Handbooks of Linguistics and Communication Science 5.4). Berlin/Boston, S. 1244-1253.

Storrer, Angelika/Freese, Katrin (1996): Wörterbücher im Internet. In: Deutsche Sprache 24, S. 97-153.

Thier, Katrin (2014): Das Oxford English Dictionary und seine Nutzer. In: Abel/Klosa (Hg),

S. 67-74.

Vossen, Piek (1998): Introduction to EuroWordNet. In: Computers and the Humanities 32, 2-3, S. 73-89. 